Research Article

\title{
Nonlinear Contour Tracking of a Voice Coil Motors-Driven Dual-Axis Positioning Stage Using Fuzzy Fractional PID Control with Variable Orders
}

\author{
Syuan-Yi Chen $\left.{ }^{1}\right)^{1}$ and Meng-Chen Yang ${ }^{2}$ \\ ${ }^{1}$ Department of Electrical Engineering, National Taiwan Normal University, Taiwan, China \\ ${ }^{2}$ Department of Hardware Design, Imagination Broadway Ltd., New Taipei City, China \\ Correspondence should be addressed to Syuan-Yi Chen; chensy@ntnu.edu.tw
}

Received 4 November 2020; Revised 1 December 2020; Accepted 2 March 2021; Published 25 March 2021

Academic Editor: Guoqiang Wang

Copyright (c) 2021 Syuan-Yi Chen and Meng-Chen Yang. This is an open access article distributed under the Creative Commons Attribution License, which permits unrestricted use, distribution, and reproduction in any medium, provided the original work is properly cited.

\begin{abstract}
This study aims to develop a variable-order fuzzy fractional proportional-integral-differential (VOFFPID) control system for controlling the mover position of a newly designed voice coil motors- (VCMs-) driven dual-axis positioning stage. First, the operation principle and dynamics of the stage are analyzed. After that, the design of a fuzzy fractional proportional-integral-differential (FFPID) control system is introduced on the basis of a fractional calculus and fuzzy logic system. With an additional degree of freedom to the control parameters and fuzzy operation, the FFPID control system can upgrade the contour tracking performance of a conventional proportional-integraldifferential (PID) control system with respect to the specified dynamics of the stage. Moreover, the VOFFPID control system is designed to further improve the tracking responses of the FFPID control system. In this system, the five control parameters are optimized with the cuckoo search algorithm via an adaptive strategy. Lastly, nominal and payload conditions attributed to two nonlinear contour demands are provided to evaluate the contouring performance of the PID, FFPID, and VOFFPID control systems. The experimental results subjected to different performance measures demonstrate that the proposed VOFFPID controller outperforms PID and FFPID controllers in terms of the designed VCMs-driven dual-axis positioning stage under both conditions.
\end{abstract}

\section{Introduction}

Although control engineers prefer a conventional proportional-integral-differential (PID) controller because of its easy implementation, low cost, and uncomplicated structure, they cannot use it to achieve a high-precision control level in a highly nonlinear and disturbed situation. To address this problem, a fractional-order (FO) PID (FPID) control method was developed by adding fractional differential and integral operations. With the consideration of more degrees of freedom for the selection of control parameters, the FPID controller can obtain better control responses and anti-interference characteristic over the integer-order (IO) counterparts because of the additional flexibility to the design of a control system [1]. However, accurately determining numerous control parameters in practical applications is difficult. Therefore, many intelligent strategies were designed for FPID control $[2,3]$.

The introduction of a fuzzy logic system (FLS) to a PID controller has been widely explored because it provides a flexible and model-free way to determine the PID control parameters through engineering intuitions and experiences $[4,5]$. In addition, fuzzy FPID (FFPID) control systems were further developed to enhance the control performances of a FPID controller $[1,6-9]$. In the FFPID, the fractional operation of errors introduces an extra degree of flexibility in the input variables of FLS, and it can be tuned similarly to the input-output scaling factors of the FLS to enhance the closed-loop performance. Some experimental results have verified that the FFPID control system outperforms classical PID, fuzzy PID, and FPID control systems because of its FLS and higher degrees of freedom for tuning. 
Swarm intelligence algorithms have been widely applied to solve many real-world problems, such as control system design [2], path planning [10], parameter estimation [11], and energy management [12], because these algorithms can obtain a global optimal solution for multidimensional optimization problems by relying on colony behaviors in nature. For example, inspired by the aggressive reproduction behavior of cuckoo bird species, cuckoo search algorithms (CSAs) were developed in [2, 13-16]. In cuckoo reproduction, female cuckoos fly from one nest to another and randomly lay their fertilized eggs inside other host birds' nests instead of building their own nests. Thus, host birds may unknowingly raise these eggs. In general, female cuckoos choose the best nest so that their eggs have the best chance of hatching and creating a new generation. To enhance the hatching chance, some cuckoo birds strategically lay their eggs in a good position or drop the host bird's eggs outside its nest. Some cuckoo species even evolve to produce eggs similar to those of other bird species. However, if an alien egg is found, host birds throw it out or even transfer to a new nest with their own brood elsewhere. In this case, the eggs of cuckoo fail to hatch. In the CSA algorithm, cuckoo birds represent the particles assigned to find the optimal solution, while cuckoo eggs and host birds' eggs represent the new and old solutions for the current iteration process, respectively. If a new solution is better than the old one, the worse one is replaced.

A linear voice coil motor (VCM) is a direct drive and hysteresis-free device, which utilizes a magnetic field generated by a permanent magnet with a coil wire to produce an electric driving force $[17,18]$. This device has a compact structure, high acceleration, and no hysteresis features, so it has been extensively used in various small range positioning applications, such as servo valves [17], hard disk drives $[19,20]$, automatic transmitters [21], autofocus actuators [22], and ultrasound scanners [23], which demand highprecision and high-speed control levels. However, effective controls for this device should be designed because external disturbances and operational changes instantly act on a direct drive system. For instance, an intelligent FO slidingmode control was proposed to control a linear voice coil actuator for the tracking of a reference trajectory [18]. In this control scheme, a fuzzy neural network was designed to compensate for system uncertainties, thereby reducing the chattering phenomena. Moreover, a coupling controller design was proposed by considering the interaction between a VCM and a piezoactuator of a head positioning control system [20]. In another study [24], a direct amplitude control strategy was developed to improve the amplitude accuracy of a reciprocating rig in a high-frequency band compared with that given by a traditional proportionalintegral control strategy.

In the direct drive VCM system, there are no mechanical reduction and transmission components so that the mover is directly coupled to the payload. Compared with the conventional rotary motor using mechanical components to translate the rotary motion into linear motion, direct drive device apparently reduces mechanical loss, system nonlinearities, and backlash [25]. Thus, the control accuracy of the
VCM system can be enhanced in practical applications. However, it also loses the advantage of using mechanical components attenuating the effects of system parameter variations and external disturbances. With this structure, the system uncertainties are directly transmitted to the payload and then unavoidably affect the control performance of the payload. On the other hand, any change or disturbance in the payload will be directly reflected back to the VCM. Although many control methods have been proposed to control the single-axis VCM systems [17, 18, 21-24], designing effective and robust control methods to meet highprecision requirements for the multi-axis VCM systems is still required. As a result, this study aims to develop a variable-order FFPID (VOFFPID) control strategy for controlling the mover position of a VCM-based dual-axis positioning stage with a high-precision contouring performance. In the VOFFPID controller, control parameters are self-tuned to deal with system uncertainty so that the trivial trials of control parameters are unnecessary. Furthermore, good stability and robustness during the control process can be ensured. Experiments involving the tracking of two nonlinear contour demands were conducted by using PID, FFPID, and VOFFPID under nominal and payload conditions to demonstrate the different control performance and robustness levels.

From the aforementioned studies, the main academic and industrial contributions of this study are summarized as follows: (i) the new VOFFPID controller that optimizes the conventional FFPID controller online is successfully developed; (ii) the new VCMs-driven dual-axis positioning stage is made with operation and dynamic analyses; (iii) the PID, FFPID, and VOFFPID controllers for the VCMs-driven dual-axis positioning stage control system are successfully implemented; and (iv) the experimental results of the three controllers associated with two nonlinear contour tracking commands under two test conditions are compared. The remaining parts of this study are organized as follows. The operation principle of VCMs-driven dual-axis positioning stage is described in Section 2. The CSA with the adaptive strategy used for optimizing the control parameters of the VOFFPID is presented in Section 3. The designs of contour tracking controllers are introduced in Section 4. The experimental setup and results are discussed in Section 5. The conclusions of the proposed work are provided in Section 6.

\section{Operation Principle of the VCMs-Driven Dual-Axis Positioning Stage}

A circular moving coil-type single-axis VCM that is composed of a moving coil winding and a stationary permanent magnet within a soft iron shell is utilized in this study as shown in Figure 1. In accordance with the interaction between the permanent magnetic field and a drive current perpendicular to the field, the mover of the VCM moves along the direction of the electric driving force, which can be determined with Fleming's left-hand rule [7]. If the direction of the drive current changes, the moving direction also reverses. Moreover, the generated electric driving force is 


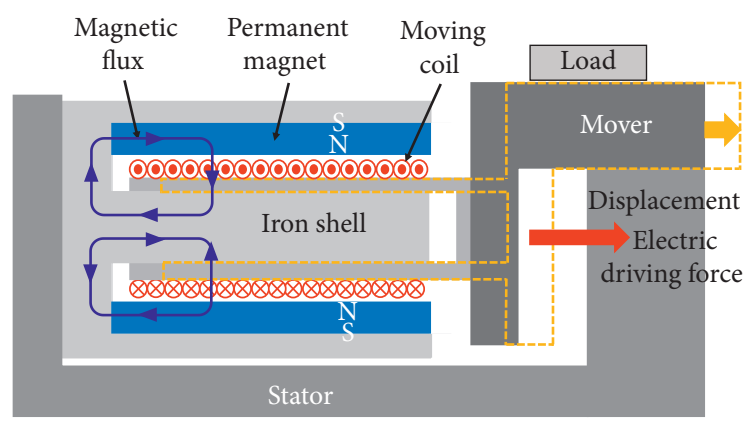

Figure 1: Structure of the VCM.

proportional to the product of the permanent magnetic field and the drive current [18].

In this study, a VCMs-driven dual-axis positioning stage is newly designed and implemented as shown in Figure 2. The dimension of the whole stage is $230 \mathrm{~mm} \times$ $194 \mathrm{~mm} \times 100 \mathrm{~mm}$. It is composed of three VCMs (Akribis, AVM 40-20), namely, a VCM in the $Y$-axis and two parallel VCMs in the $X$-axis. A $100 \mathrm{~mm}^{2}$ moving platform is placed on the mover of the $Y$-axis VCM, and the stator of the $Y$-axis VCM is mounted on a moving base. With the design of this stage, two VCMs in the $X$ axis can generate a stronger electromagnetic force to push the moving platform, moving base, $Y$-axis VCM, and payload along the $X$-axis. They can even create rotational motion according to the specified mechanism design and different displacements of $X$-axis VCMs. Two high-resolution linear scales measure the mover displacements for high-precision and repeatability applications. Specifications of the adopted VCMs are listed in Table 1 [26].

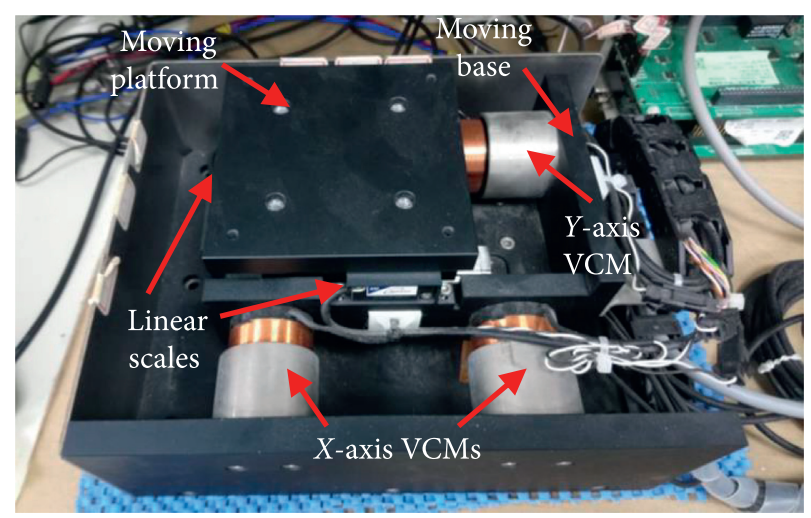

FIgURE 2: Structure of the VCMs-driven dual-axis positioning stage.

TABLe 1: Specifications of the adopted VCMs.

\begin{tabular}{lcc}
\hline Specifications & Value & Unit \\
\hline Diameter & 40 & $\mathrm{~mm}$ \\
Stroke & 20 & $\mathrm{~mm}$ \\
Force-current coefficient & 12.90 & $\mathrm{~N} / \mathrm{A}$ \\
Back electromotive force constant & 12.90 & $\mathrm{~V} / \mathrm{m} / \mathrm{s}$ \\
Continuous force & 9.93 & $\mathrm{~N}$ \\
Peak force & 58.05 & $\mathrm{~N}$ \\
Continuous current & 0.77 & $\mathrm{~A}$ \\
Peak current & 4.5 & $\mathrm{~A}$ \\
Continuous power & 7.17 & $\mathrm{~W}$ \\
Coil assembly mass & 67.0 & $\mathrm{~g}$ \\
Core assembly mass & 226.2 & $\mathrm{~g}$ \\
\hline
\end{tabular}

The state-space model is given below to describe the dynamics of the VCMs-driven dual-axis positioning stage [17]:

$$
\begin{aligned}
& \left\{\ddot{x}=-\frac{k_{k x}+\Delta k_{k x}}{m_{b}+m_{p}+m_{l}} x-\frac{k_{b x}+\Delta k_{b x}}{m_{b}+m_{p}+m_{l}} \dot{x}+\frac{c_{x} k_{i x}+\Delta c_{x} k_{i x}}{m_{b}+m_{p}+m_{l}} u_{x}-\frac{F_{f x}+F_{\mathrm{d} x}}{m_{b}+m_{p}+m_{l}},\right. \\
& \ddot{y}=-\frac{k_{k y}+\Delta k_{k y}}{m_{p}+m_{l}} y-\frac{k_{b y}+\Delta k_{b y}}{m_{p}+m_{l}} \dot{y}+\frac{c_{y} k_{i y}+\Delta c_{y} k_{i y}}{m_{p}+m_{l}} u_{y}-\frac{F_{f y}+F_{\mathrm{d} y}}{m_{p}+m_{l}},
\end{aligned}
$$

where $x$ and $y$ are the mover positions in $X$-axis and $Y$-axis, respectively; $u_{x}$ and $u_{y}$ indicate the control signals of the VCMs; $c_{x}$ and $c_{y}$ represent the linear gains of the current amplifiers; $k_{i x}$ and $k_{i y}$ are the force-current coefficients of the VCMs; $k_{b x}$ and $k_{b y}$ are the equivalent damping coefficients; $k_{k x}$ and $k_{k y}$ denote the equivalent elastic coefficients; $\Delta k_{k x}$, $\Delta k_{k y}, \Delta k_{b x}, \Delta k_{b y}, \Delta c_{x} k_{i x}$, and $\Delta c_{y} k_{i y}$ represent the unknown parameter variations of $k_{k x}, k_{k y}, k_{b x}, k_{b y}, c_{x} k_{i x}$, and $c_{y} k_{i y}$, respectively; $m_{b}, m_{p}$, and $m_{l}$ denote the masses of the moving base, platform, and payload, respectively; $F_{f x}$ and $F_{f y}$ are the friction forces; and $F_{d x}$ and $F_{d y}$ denote the unmodeled system uncertainties, comprising internal cross-coupled interferences and external disturbances. Thus, the dynamic model of the VCMs-driven dual-axis positioning stage can be reexpressed as

$$
\left\{\ddot{x}=-\frac{k_{k x}}{M_{x}} x-\frac{k_{b x}}{M_{x}} \dot{x}+\frac{c_{x} k_{i x}}{M_{x}} u_{x}-\frac{L_{x}}{M_{x}}, \ddot{y}=-\frac{k_{k y}}{M_{y}} y-\frac{k_{b y}}{M_{y}} \dot{y}+\frac{c_{y} k_{i y}}{M_{y}} u_{y}-\frac{L_{y}}{M_{y}},\right.
$$


where $M_{x}=m_{b}+m_{p}+m_{l}$ and $M_{y}=m_{p}+m_{l} ; L_{x}$ and $L_{y}$ are the lumped uncertainties regarded as follows:

$$
\left\{\begin{array}{l}
L_{x}=\Delta k_{k x} x+\Delta k_{b x} \dot{x}-\Delta c_{x} k_{i x} u_{x}+F_{f x}+F_{d x} \\
L_{y}=\Delta k_{k y} y+\Delta k_{b y} \dot{y}-\Delta c_{y} k_{i y} u_{y}+F_{f y}+F_{d y}
\end{array}\right.
$$

In equation (1), the practical control characteristics of the VCMs are nonlinear because the system coefficients described above may vary due to the changes in operating temperature and duration, though the VCMs-driven dual-axis positioning stage can be presented with a state-space model. Moreover, the lumped uncertainties $L_{x}$ and $L_{y}$ cannot be measured exactly. Therefore, designing a model-free control method is important to control the VCMs-driven dual-axis positioning stage with a stable and precise nonlinear contour tracking performance for the practical applications.

\section{CSA with an Adaptive Strategy}

CSA is a metaheuristic evolutionary algorithm based on the aggressive reproduction of a cuckoo species with a Lévy flight behavior. Three idealized characteristic rules are assumed as follows to formulate the CSA [13-16]:

(a) Each cuckoo bird lays one egg in a randomly selected host nest, representing a solution to the optimization problem.

(b) Some of these nests contain high-quality eggs, representing good solutions, which are preserved for the next generation.

(c) The number of available host nests is fixed in the ecosystem, and the probability of alien eggs discovered by the host bird is $P_{a} \in[0,1]$. When the host bird finds the alien eggs, it destroys the egg or abandons the old nest and builds a new one in another place.

3.1. Principle of CSA. From the optimization perspective, cuckoo birds correspond to the particles assigned to find solutions, and cuckoo eggs indicate the candidate solutions for an optimization problem. In the CSA, the random step of cuckoo birds is characterized by a Lévy flight, indicating that the step length of the flight behavior follows the Lévy distribution; consequently, the CSA realizes a "random walk" and a "long jump" among their flights [15]. In this regard, the CSA can avoid obtaining an unreliable local optimal solution and shorten the convergence time required to reach a global optimal solution.

An unconstrained optimization problem can be stated as follows:

$$
\text { find } \mathbf{x}=\left[x_{1}, x_{2}, \ldots, x_{D}\right], \quad \text { which maximizes } J(\mathbf{x}),
$$

where $\mathbf{x}$ is the individual nest position, $D$ is the optimized variable dimension, and $J$ is an objective function. In the CSA, the update of the egg position is given according to a Lévy flight as follows [13-16]:

$$
\mathbf{x}_{i, k+1}=\mathbf{x}_{i, k}+\alpha \oplus \operatorname{Lévy}(\beta),
$$

where $i=1,2, \ldots, N_{p}$ is the population size, $k$ is the current index for the generation iteration, $\oplus$ is entry-wise multiplication, $\alpha>0$ is a step size related to the scales of the problem of interest, and $1 \leq \beta \leq 3$ is a parameter used to formulate the Lévy distribution and it is considered to be 1.5 in this study. Then, the step length $\varsigma$ is defined as

$$
\varsigma=\frac{\mu}{|v|^{(1 / \beta)}},
$$

where $\mu$ and $v$ are random numbers derived from normal distribution as

$$
\begin{aligned}
& \mu \sim N\left(0, \sigma_{\mu}^{2}\right), \\
& v \sim N\left(0, \sigma_{v}^{2}\right), \\
& \sigma_{\mu}=\left\{\frac{\Gamma(1+\beta) \times \sin (\pi \beta / 2)}{\Gamma[(1+\beta) / 2] \times \beta \times 2^{(\beta-1) / 2}}\right\}^{(1 / \beta)}, \\
& \sigma_{v}=1 .
\end{aligned}
$$

where $\sigma_{\mu}$ is derived by using Mantegna's algorithm for symmetric distributions and $\Gamma(\cdot)$ is a Gamma function. Then, the step size $\mathbf{s}$ is calculated as

$$
\mathbf{s}_{i, k}=\alpha \cdot \varsigma \cdot\left(\mathbf{x}_{i, k}-\mathbf{x}_{b}\right)
$$

where $\mathbf{x}_{b}$ is the current best solution. Thus, the update of the egg position as shown in equation (5) can be formulated:

$$
\mathbf{x}_{i, k+1}=\mathbf{x}_{i, k}+r \cdot \mathbf{s}_{i, k},
$$

where $r$ is a random value following the normal distribution $N(0,1)$. Figure 3 shows the typical trajectory of a threedimension random Lévy flight path by using equations (5)-(10). Afterward, the fitness values of $J\left(\mathbf{x}_{i, k+1}\right)$ and $J\left(\mathbf{x}_{i, k}\right)$ are compared. If $J\left(\mathbf{x}_{i, k+1}\right)>J\left(\mathbf{x}_{i, k}\right)$ holds, the $i^{\text {th }}$ solution is replaced, and the new solution is accepted as $\mathbf{x}_{i, k+1}$. In addition, the parameter $P_{\theta}$ is set as the threshold of discovery probability that the cuckoo's eggs are found by a host bird. The host bird builds nests at new locations according to

$$
\mathbf{x}_{i, k+1}= \begin{cases}\mathbf{x}_{i, k}+r \cdot\left(\mathbf{x}_{q, k}-\mathbf{x}_{j, k}\right), & \text { if } P>P_{\theta}, \\ \mathbf{x}_{i, k}, & \text { else }\end{cases}
$$

where $\mathbf{x}_{q, k}$ and $\mathbf{x}_{j, k}$ are two randomly selected different solutions in the $k^{\text {th }}$ iteration and $P$ is a uniform random number distributed in $[0,1]$. Similarly, if the fitness value of the new solution is better than the old one, then the new solution $\mathbf{x}_{i, k+1}$ is used to replace the old one $\mathbf{x}_{i, k}$.

3.2. Adaptive Strategy of the CSA. An adaptive strategy based on Rechenberg's $1 / 5$ criteria is utilized to enhance the evolution and adaptation efficiency of the CSA [14]. With the adaptive strategy, step size and discovery probability are dynamically tuned during optimization. First, the improvement rate $\zeta$ is defined as follows:

$$
\zeta=\frac{N_{r}}{N_{p}}
$$

where $N_{r}$ is the number of all cuckoo birds whose fitness values are improved after evolution. Thus, the step size $\alpha$ and discovery probability $P_{\theta}$ can be further updated as 


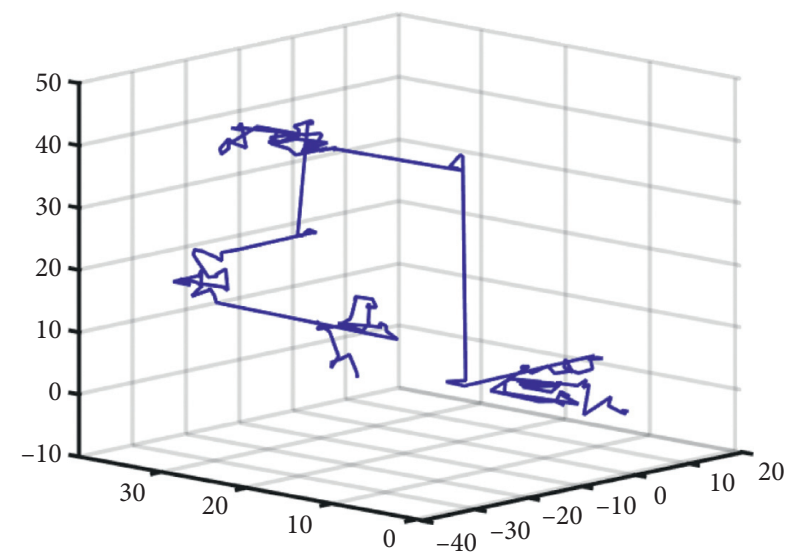

FIgURE 3: Typical trajectory of a three-dimension random Lévy flight path.

$$
\begin{gathered}
\alpha_{k+1}= \begin{cases}\alpha_{k} \times f_{\alpha}, & \zeta>\alpha_{u}, \\
\alpha_{k}, & \alpha_{l} \leq \zeta \leq \alpha_{u}, \\
\frac{\alpha_{k}}{f_{\alpha}}, & \zeta<\alpha_{l},\end{cases} \\
P_{\theta, k+1}= \begin{cases}P_{\theta, k} \times f_{p}, & \zeta>P_{u}, \\
P_{\theta, k}, & P_{l} \leq \zeta \leq P_{u}, \\
\frac{P_{\theta, k},}{f_{p}} & \zeta<P_{l},\end{cases}
\end{gathered}
$$

where $\alpha_{u}$ and $\alpha_{l}$ are the upper and lower thresholds of $\zeta$ with respect to $\alpha ; P_{u}$ and $P_{l}$ are the upper and lower thresholds of $\zeta$ with respect to the discovery probability; and $1 \leq f_{\alpha} \leq 2$ and $1 \leq f_{P} \leq 2$ are the learning factors of $\alpha$ and $P_{\theta}$, respectively.

According to equations (13) and (14), $\alpha$ and $P_{\theta}$ are increased to strengthen the global exploration ability when $\zeta$ is large. This result indicates that the current solution space is relatively monotonous and smooth. On the contrary, $\alpha$ and $P_{\theta}$ are decreased to enhance the local exploitation ability when $\zeta$ is small. This result suggests that the optimal solution may be in the surrounding search area near the current solution. In this regard, the local exploitation and global exploration abilities of the CSA can be well balanced to deal with the diversification and intensification of a population, thereby avoiding the premature convergence.

\section{Control System Designs of the VCMs-Driven Dual-Axis Positioning Stage}

First, typical PID and FFPID control strategies are adopted in this study to control the VCMs-driven dual-axis positioning stage for nonlinear contour tracking. Subsequently, a VOFFPID is proposed to improve the stability and accuracy of the contour tracking performance under system uncertainties, including parameter variations, cross-coupled interferences, and friction forces [27]. With the help of onlinetuned control parameters, the system uncertainties can be compensated, and the high-precision nonlinear contour tracking performance can be guaranteed.
4.1. Typical PID Control. The popularity of IO PID (IOPID) controllers as expressed in equation (15) can be attributed partly to their favorable performance in a wide range of applicability and partly to their functional simplicity, which allows engineers to operate them in an easy and straightforward manner. As for PID controller, the proportional (P) action amplifies errors, the integral (I) action accumulates errors, and the differential (D) action calculates the change in errors. In this study, the PID controller compares the actual mover positions $x$ and $y$ with the reference contour positions $x_{d}$ and $y_{d}$ to obtain the error signals $e_{x}$ and $e_{y}$. After that, it accumulates the results of the P, I, and D actions as below [7]:

$$
u_{j}(t)=K_{\mathrm{Pj}} e_{j}(t)+K_{\mathrm{I} j} \int_{0}^{t} e_{j}(\tau) \mathrm{d} \tau+K_{\mathrm{D} j} \frac{\mathrm{d}}{\mathrm{d} t} e_{j}(t)
$$

where $t$ denotes the current time; $j=x, y$ represent the $X$-axis and $Y$-axis of the VCMs-driven dual-axis positioning stage, respectively; $u_{x}$ and $u_{y}$ denote the control signals; $K_{\mathrm{P} j}, K_{\mathrm{I} j}$, and $K_{\mathrm{D} j}$ denote the $\mathrm{P}, \mathrm{I}$, and $\mathrm{D}$ control parameters, respectively; and $e_{x}$ and $e_{y}$ indicate the tracking errors defined as $e_{x}=x_{d}-x$ and $e_{y}=y_{d}-y$, respectively.

4.2. FO Integral and Differential Definitions. FO integral and differential operators are defined in the following $[7,18,25]$ :

$$
{ }_{a} D_{t}^{\lambda}= \begin{cases}\frac{\mathrm{d}^{\lambda}}{\mathrm{d} t^{\lambda},} & \lambda>0, \\ 1, & \lambda=0, \\ \int_{a}^{t}(\mathrm{~d} \tau)^{-\lambda}, & \lambda<0,\end{cases}
$$

in which $D$ is the fractional calculus operator; $\lambda$ is the fractional order; and $a$ and $t$ represent the operation range. The three major FO integral and differential definitions are the Caputo, Grunwald-Letnikov (GL), and Riemann-Liouville (RL) definitions. The operator given in equation (16) applied to the $f(t)$ function leads to an extended Caputo form, which can be derived as follows [28, 29]:

tcusts 650

$$
{ }_{a} D_{t}^{\lambda} f(t)=\frac{1}{\Gamma(m-\lambda)} \int_{a}^{t} \frac{f^{(m)}(\tau)}{(t-\tau)^{\lambda+1-m}} \mathrm{~d} \tau, \quad m-1 \leq \lambda<m,
$$

where $m$ is an integer such that $m>\lambda$. Moreover, the $\lambda^{\text {th }}$ order RL FO integral of $f(t)$ is defined as follows [30]:

$$
{ }_{a} D_{t}^{-\lambda} f(t)=\frac{1}{\Gamma(\lambda)} \int_{a}^{t}(t-\tau)^{\lambda-1} f(\tau) \mathrm{d} \tau .
$$

Similarly, the RL FO differential of $f(t)$ is defined as

$$
{ }_{a} D_{t}^{\lambda} f(t)=\frac{1}{\Gamma(m-\lambda)} \frac{\mathrm{d}^{m}}{\mathrm{~d} t^{m}} \int_{a}^{t} \frac{f(\tau)}{(t-\tau)^{\lambda+1-m}} \mathrm{~d} \tau .
$$

By contrast, the $\lambda^{\text {th }}$-order GL FO operation based on finite differences is defined as follows [29]:

$$
{ }_{a} D_{t}^{\lambda} f(t)=\lim _{h \longrightarrow 0} h^{-h} \sum_{j=0}^{[t-a / h]}(-1)^{j}\left(\begin{array}{l}
\lambda \\
j
\end{array}\right) f(t-j h),
$$


where [.] is the integer part, $h$ is the time increment, and () is the fractional binomial coefficient defined as

$$
\left(\begin{array}{l}
\lambda \\
j
\end{array}\right)=\frac{\Gamma(\lambda+1)}{\Gamma(j+1) \cdot \Gamma(\lambda-j+1)} .
$$

Intuitively, integral and differential operations with fractional orders can provide a higher degree of freedom to the control parameters than those with integer orders. As a result, the control performance of PID control system can be enhanced by properly selecting fractional integral and differential orders. For convenience, the FO operator ${ }_{a} D_{t}^{\lambda}$ is noted as $D^{\lambda}$ in the subsequent sections.

4.3. Developed FFPID Control System. In the case of a nonlinear and disturbed system, the conventional IOPID control strategy is difficult to concurrently obtain a high control performance level and maintain good robustness because of its linear structure [7]. To improve the control performances, smoothness and robustness of the PID control system, the FFPID control, which combines the merits of PID control, FO operations, and FLS, is adopted and illustrated in Figure 4 in this study. In Figure $4, a_{j}$ and $b_{j}$ are the fractional differential and integral orders, respectively; $K_{\mathrm{P} j}$ and $K_{\mathrm{D} j}$ can be considered the input scaling factors; and $K_{\mathrm{I} j}$ can be regarded as the output scaling factor. The inputs of the FLS are the tracking error $e_{j}$ multiplied by $K_{\mathrm{P} j}$ and the fractional differential of the tracking error $D^{a j} e_{j}$ multiplied by $K_{\mathrm{D} j}$, which can be regarded as a $\mathrm{FO}$ proportional-differential (FOPD) controller. The relationship between the inputs and output of the FLS is specified with the table of the fuzzy rules as given in Table 2 in which the fuzzy linguistic values NL, NM, NS, ZO, PS, PM, and PL indicate negatively large, negatively medium, negatively small, zero, positively small, positively medium, and positively large, respectively [7]. Figure 5 illustrates the membership functions for the inputs and output of FLS in which the horizontal range was designed on the basis of the prior experimental tests to effectively cover the input and output signals [7]. In this study, the triangular membership functions, which can be easily configured with regard to the linear shape and fewer parameters, were selected to ease the computational burden and speed up the control process. Thus, the output of the FLS $u_{\mathrm{FPD} j}$ can be derived according to the designed fuzzy rules with the center of gravity defuzzification method as follows:

$$
u_{\mathrm{FPD} j}=\frac{\sum_{k=1}^{n} \mu_{c}\left(\sigma_{k}\right) \sigma_{k}}{\sum_{k=1}^{n} \sigma_{k}},
$$

where $c$ indicates a logical union set of the conclusion fuzzy sets of the fired fuzzy rules; $\sigma_{k}$ is a value between the minimum and maximum values of the abscissa of $c$ defined on the universe of discourse; $\mu_{c}\left(\sigma_{k}\right)$ is the firing strength of $c$ for the point $\sigma_{k}$; and $n$ is the number of the samples.

The final control signal of the FFPID control system $u_{j}$ is the sum of the output of FLS $u_{\mathrm{FPD} j}$ multiplied by $\lambda_{j}$ and the fractional integral of the output of FLS $u_{\mathrm{FPD} j}$ multiplied by $K_{\mathrm{I} j}$ :

$$
u_{j}(t)=\lambda_{j} u_{\mathrm{FPD} j}(t)+K_{\mathrm{I} j} D^{-b j} u_{\mathrm{FPD} j}(t) .
$$

In equations (22) and (23), the whole FFPID controller can be considered a combination of the fuzzy FOPD controller $u_{\mathrm{FPD} j}$ in the first half and the FO proportional-integral (FOPI) controller $u_{j}$ in the second half. The benefits of the FFPID controller are adjustability and flexibility when these two controllers are combined. On the other hand, as seen from Figure 4, the integral operator $D^{-b j} e_{j}$ can be regarded a low-pass filter of the error signal $e_{j}$. When $b_{j}$ is appropriately selected, the steady-state error can be suppressed effectively [18]. Besides, the differential operator $D^{a j} e_{j}$ can be regarded a high-pass filter of $e_{j}$. A proper $a_{j}$ can accelerate the dynamic response of the VCMs-driven dual-axis positioning system. Therefore, the contour tracking responses with a conventional IOPID controller can be enhanced by adding the welldefined fractional orders $a_{j}$ and $b_{j}$ regarding the specified dynamics of the VCMs-driven dual-axis positioning stage.

4.4. Proposed VOFFPID Control System. The control gains (i.e., $K_{\mathrm{P} j}, K_{I j}$, and $K_{D j}$ ), along with fractional orders of differentiation (i.e., $a_{j}$ ) and integration (i.e., $b_{j}$ ), are tuned to obtain the optimum contour tracking performance of the VCMs-driven dual-axis positioning system. Hence, a VOFFPID controller is further proposed, in which the control parameters $\left\{K_{\mathrm{P} j}, K_{\mathrm{I} j}, K_{\mathrm{D} j}, a_{j}, b_{j}\right\}$ are dynamically tuned with the CSA with an adaptive strategy. In the CSA application, the most crucial step is to choose the objective function for evaluating the fitness value of each host nest. In this study, an absolute tracking error is employed to design the objective function. Thus, the optimization problem arising in this study can be expressed by rewriting equation (4) as follows:

$$
\text { Find } \mathbf{x}=\left[K_{\mathrm{P} j}, K_{\mathrm{I} j}, K_{\mathrm{D} j}, a_{j}, b_{j}\right], \quad \text { which maximizes } J(\mathbf{x})=\frac{1}{\varepsilon+\left|e_{c}(\mathbf{x})\right|},
$$

where $\varepsilon$ is a small positive constant and $e_{c}$ is a contour tracking error defined as follows:

$$
e_{c}(\mathbf{x})=\sqrt{e_{x}(\mathbf{x})^{2}+e_{y}(\mathbf{x})^{2}} .
$$

According to the design of the object function shown in equation (24), $K_{\mathrm{P} j}, K_{I j}, K_{D j}, a_{j}$, and $b_{j}$ can be updated dynamically to minimize the contour tracking error $e_{c}$ via the CSA.

In the beginning of the VOFFPID control system, several nest positions $\mathbf{x}$ are selected randomly within the specific searching ranges. Then, each vector $\mathbf{x}$ is sequentially applied to the VOFFPID controller, and the corresponding tracking performance is evaluated via the object function $J$. Lastly, the vector with the highest fitness value is selected for the VCMs-driven dual-axis positioning system. As a result, the VOFFPID controller can achieve favorable robustness against uncertainties and external disturbances.

\section{Experimental Results}

5.1. Experimental Setup. Figure 6 shows the experimental setup of the VCMs-driven dual-axis positioning system, which consists of a newly developed dual-axis positioning stage, power supplies, servo drivers (Elmo Cello 5/60), and a 


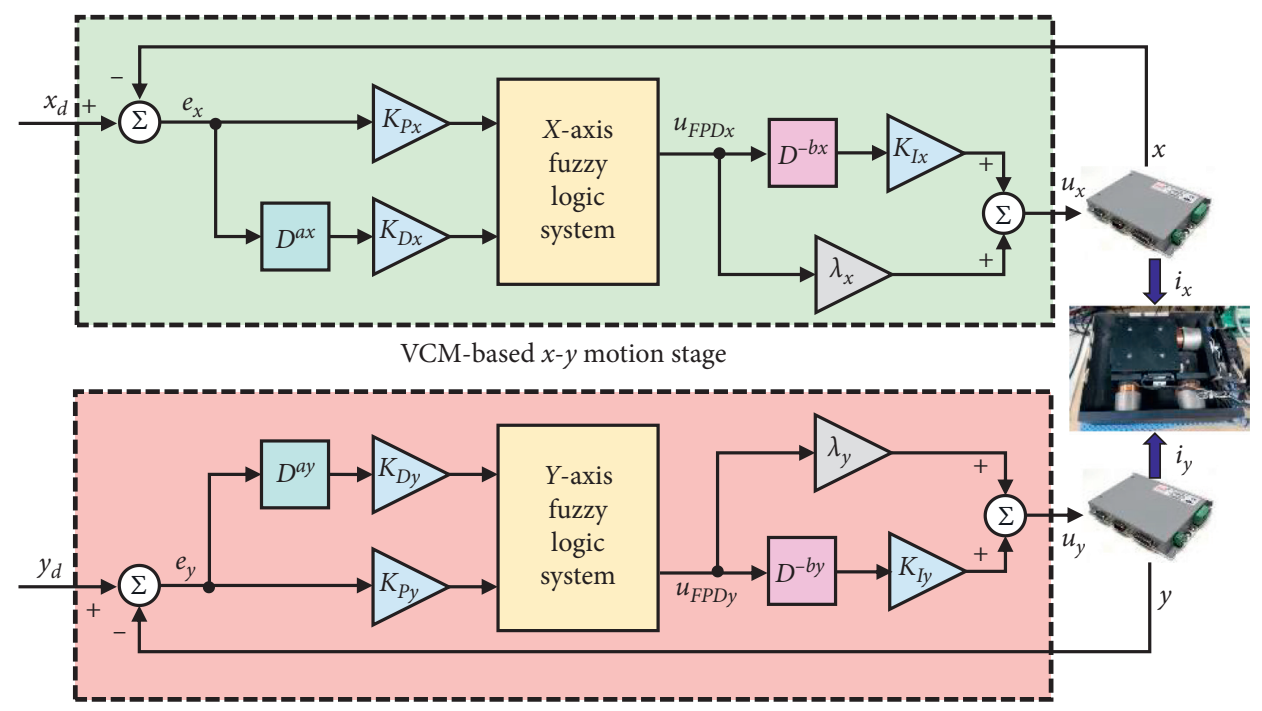

FIgURE 4: Control diagram of the VCMs-driven dual-axis positioning control system using FFPID controller.

TABLE 2: Fuzzy rule table.

\begin{tabular}{|c|c|c|c|c|c|c|c|}
\hline$K_{D j} D^{a j} e_{j} K_{p j} e_{j}$ & NL & NM & NS & ZR & PS & $\mathrm{PM}$ & PL \\
\hline PL & $\mathrm{ZR}$ & PS & $\mathrm{PM}$ & PL & PL & PL & PL \\
\hline $\mathrm{PM}$ & NS & ZR & PS & $\mathrm{PM}$ & PL & PL & PL \\
\hline PS & NM & NS & ZR & PS & $\mathrm{PM}$ & PL & PL \\
\hline ZR & NL & NM & NS & ZR & PS & $\mathrm{PM}$ & PL \\
\hline NS & NL & $\mathrm{NL}$ & NM & NS & $\mathrm{ZR}$ & PS & $\mathrm{PM}$ \\
\hline NM & $\mathrm{NL}$ & $\mathrm{NL}$ & NL & NM & NS & ZR & PS \\
\hline NL & NL & NL & NL & NL & NM & NS & ZR \\
\hline
\end{tabular}

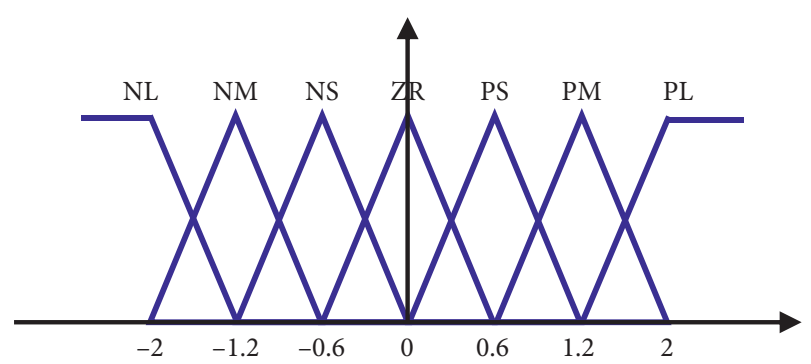

FIgURE 5: Membership functions of the input and output variables of FLS.

TMS320F28377 digital signal processor (DSP; Texas Instruments) [7]. The real-time control software developed in the DSP comprises one main program and one interrupt service routine (ISR). In the main program, parameters and I/O initializations are initially established, and the interrupt interval for the ISR is set. When the interrupt is enabled, the ISR, with $1 \mathrm{~ms}$ execution frequency, calculates the mover position from the encoder interfaces and then determines the control signals through the designed PID, FFPID, and VOFFPID control systems. After that, the control signals are sent to the servo drivers via the 14-bit resolution digital-to-analog

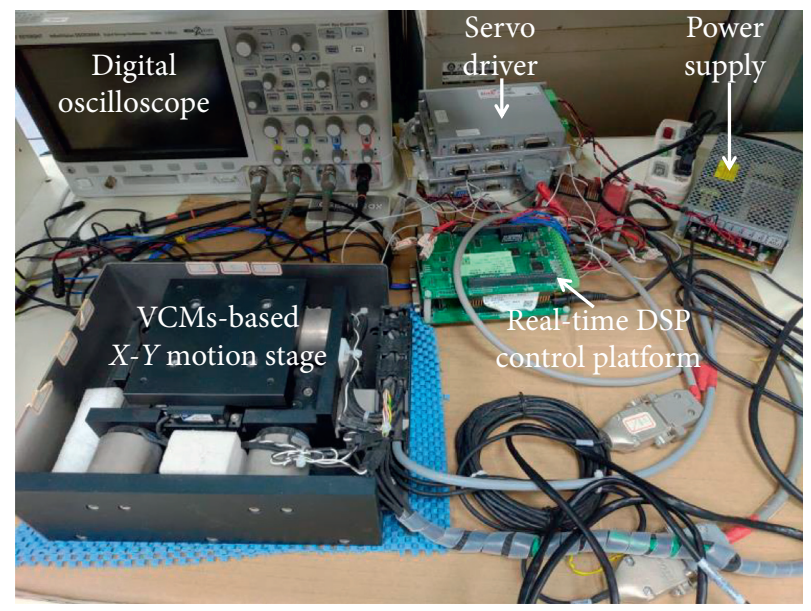

Figure 6: Experiment setup of the VCMs-driven dual-axis positioning stage.

converters (DACs) of the DSP. Afterward, the servo drivers convert the control signals to drive currents so that the VCMs can produce the required thrust force for high-precision contour tracking. In this study, a flower contour and a window contour are designed for the reference nonlinear contour commands as shown in Figures 7 and 8, respectively. 


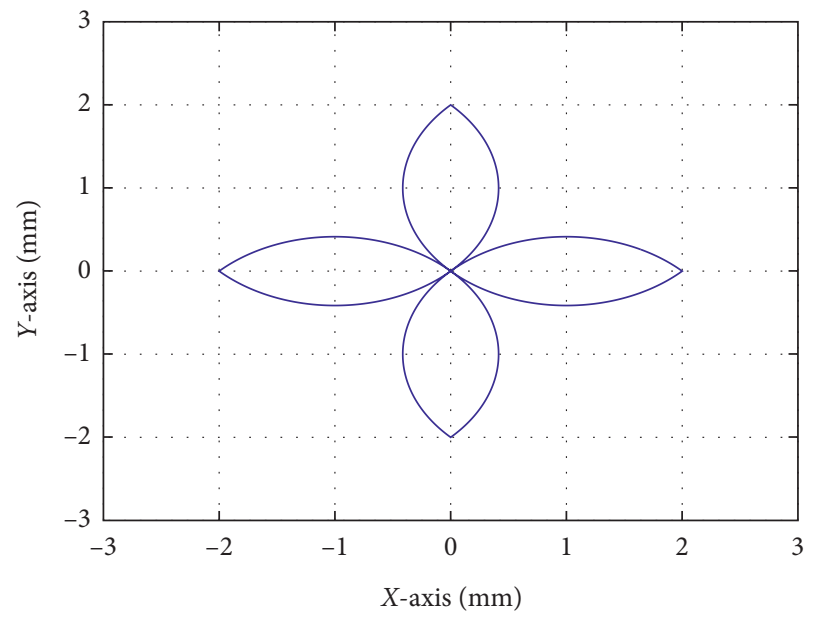

_ Flower contour command
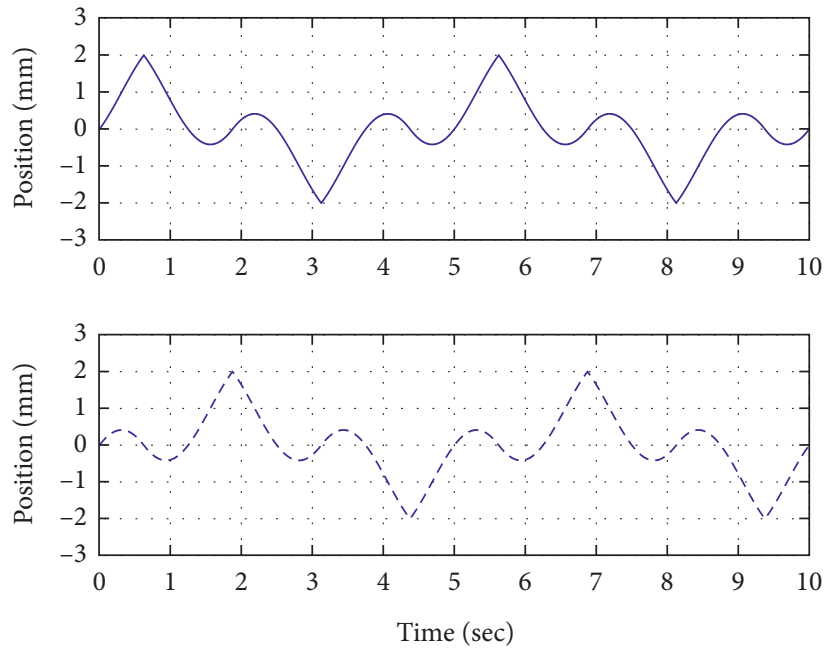

- $X$-axis mover command

- - $Y$-axis mover command

(a)

(b)

FiguRe 7: Design of flower contour command. (a) Flower contour in $X$ - $Y$-axes; (b) mover commands of flower contour in $X$-axis and $Y$-axis.

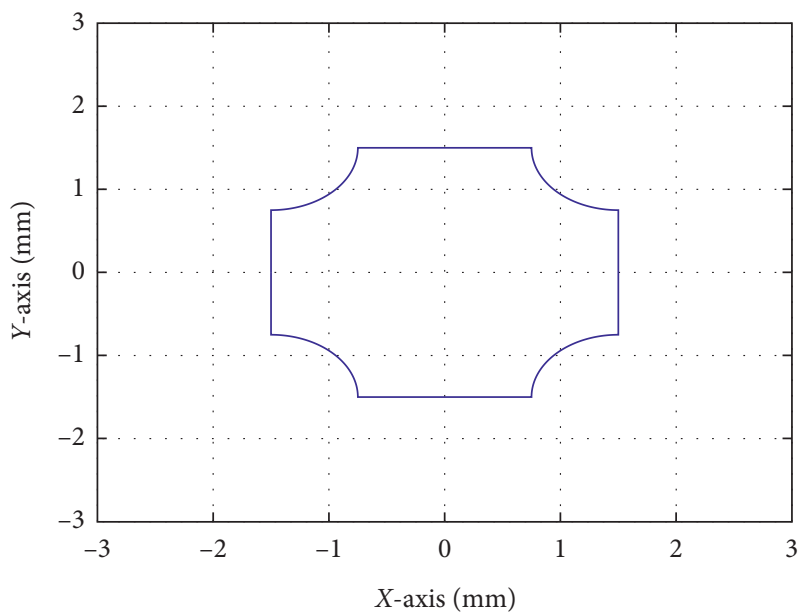

— Window contour command
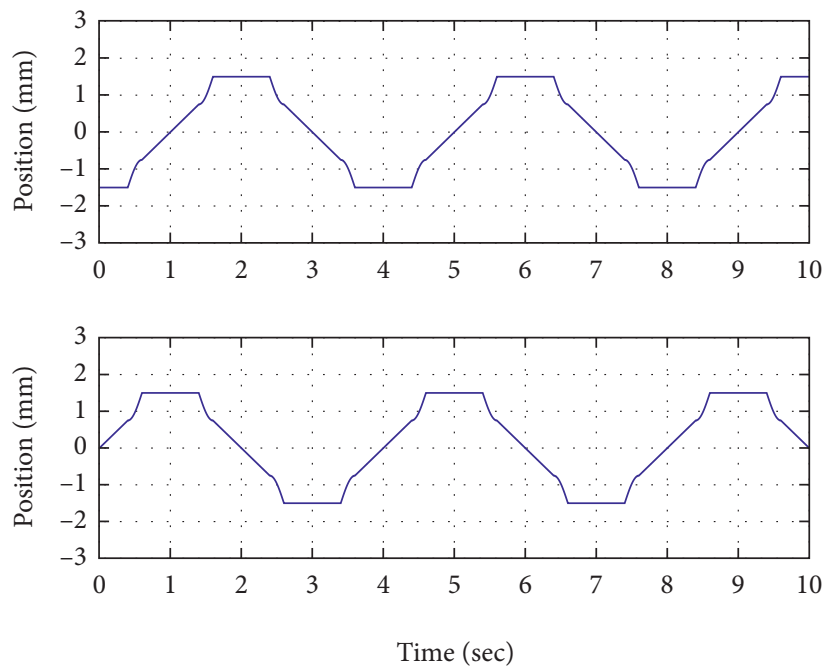

- $X$-axis mover command

— Y-axis mover command

(b)

Figure 8: Design of window contour command. (a) Window contour in $X$ - $Y$-axes; (b) mover commands of window contour in $X$-axis and $Y$-axis.

TABle 3: Formulas $A_{n}\left(z^{-1}, \lambda\right)$ for $n=0,1,3$, and 5 .

\begin{tabular}{lc}
\hline$n$ & $A_{n}\left(z^{-1}, \lambda\right)$ \\
\hline 0 & 1 \\
1 & $-\lambda z^{-1}+1$ \\
3 & $-(1 / 3) \lambda z^{-3}+(1 / 3) \lambda^{2} z^{-2}-\lambda z^{-1}+1$ \\
5 & $-(1 / 5) \lambda z^{-5}+(1 / 5) \lambda^{2} z^{-4}-\left((1 / 3) \lambda+(1 / 15) \lambda^{3}\right) z^{-3}+(2 / 5) \lambda^{2} z^{-2}-\lambda z^{-1}+1$ \\
\hline
\end{tabular}



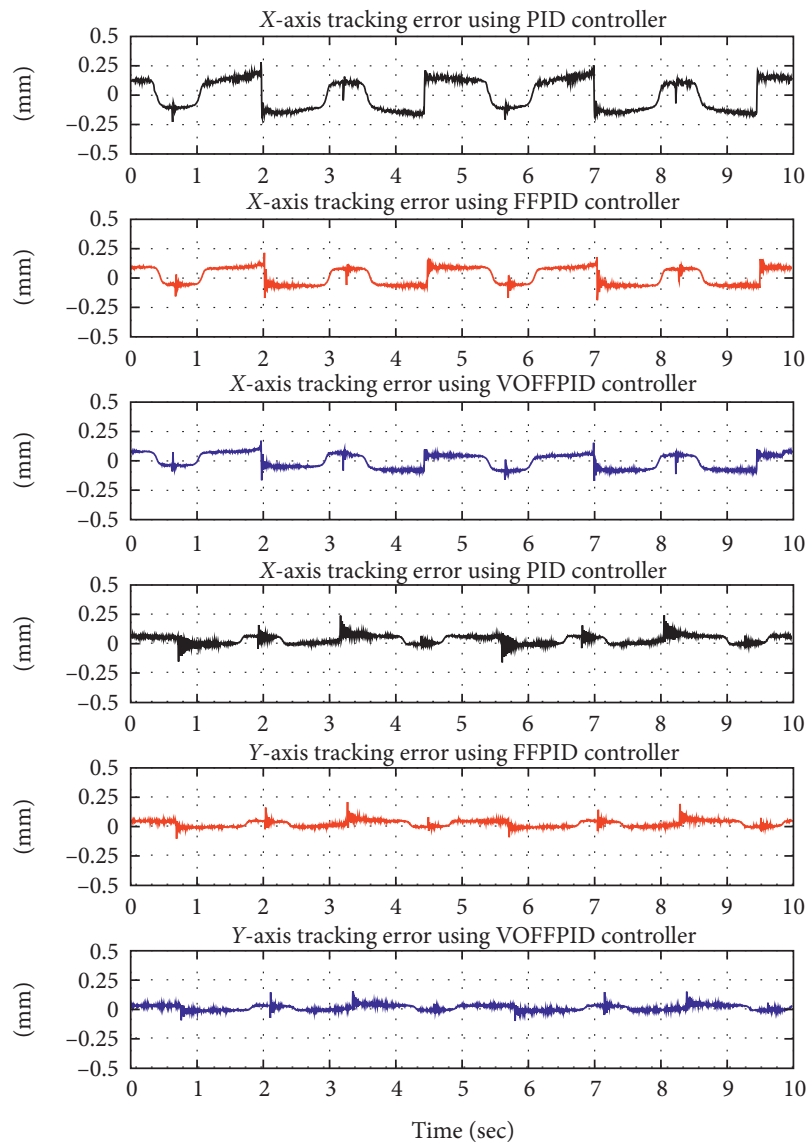

(a)
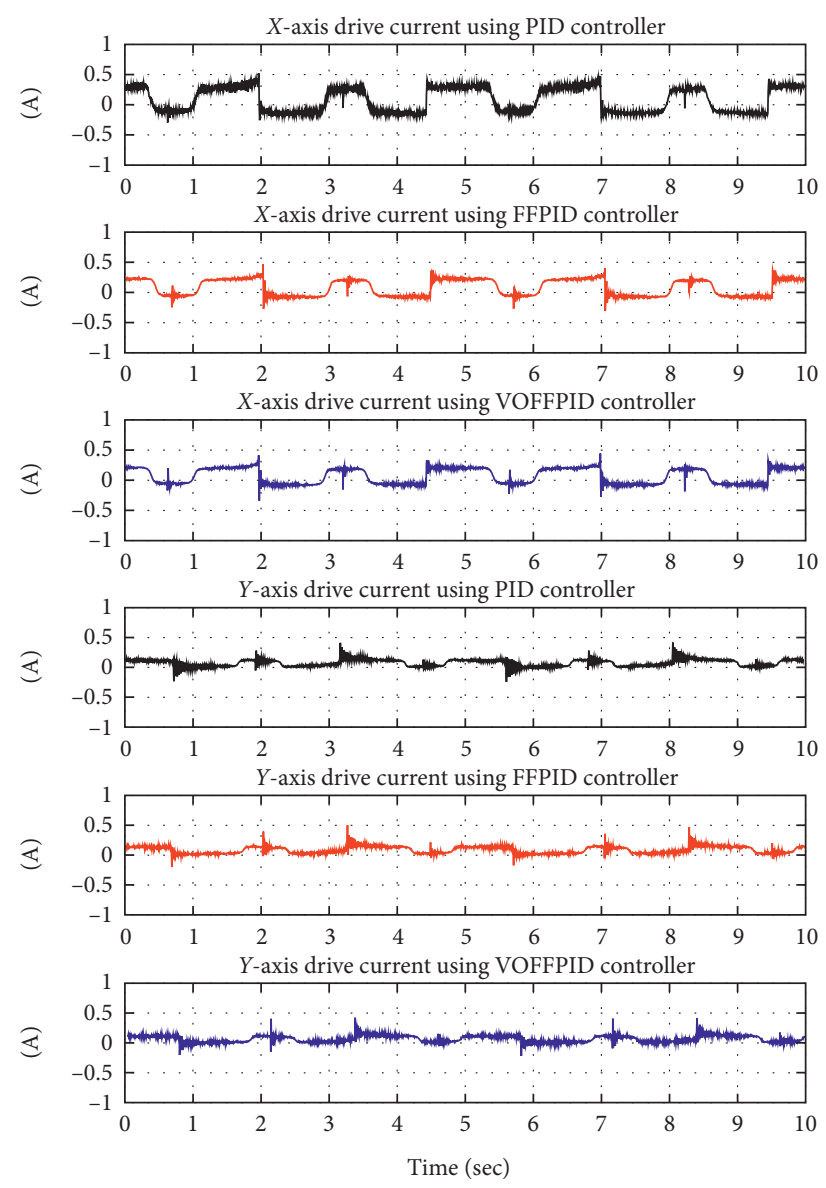

(b)

FIGURE 9: Flower contour tracking results of the VCMs-driven dual-axis positioning stage using PID, FFPID, and VOFFPID controllers in Case 1. (a) Tracking errors; (b) drive currents.

The maximum, average, and standard deviation of the contour tracking error $T_{m}, T_{A}$, and $T_{S}$ are measured as follows to compare the different positioning performance levels of the PID, FFPID, and VOFFPID control systems [7]:

$$
\begin{aligned}
T_{M} & =\max _{I} e_{c}(I), \\
T_{A} & =\sum_{I=1}^{N_{T}} \frac{e_{c}(I)}{N_{T}}, \\
T_{S} & =\sqrt{\sum_{I=1}^{N_{T}} \frac{\left[e_{c}(I)-T_{A}\right]^{2}}{N_{T}}},
\end{aligned}
$$

where $I$ is the current iteration number and $N_{T}$ is the total number of iterations. Moreover, two conditions are tested in this study: nominal (Case 1) and payload (Case 2) cases. In Case 2, one payload with a $5 \mathrm{~kg}$ weight is added to the mover.

5.2. Discretization of FO Integral and Differential. As seen from the FO definitions shown in (17)-(20), the Laplace transform of the FO differential and integral of function $f(t)$ can be represented by $s^{\lambda} F(s)$, where $s=j \omega$ is the Laplace transform operator. Hence, the Tustin method is used to obtain the coefficients and the form of the direct discretization of $s^{\lambda}$. To simplify the presentation, only the recursive formula for a positive $\lambda$ is considered. Thus, the continuous Laplace operator can be replaced by a generating function as follows $[18,25]$ :

$$
s^{\lambda}=\left(\omega\left(z^{-1}\right)\right)^{\lambda}=\left(\frac{2}{T}\right)^{\lambda}\left(\frac{1-z^{-1}}{1+z^{-1}}\right)^{\lambda}=\left(\frac{2}{T}\right)^{\lambda} \lim _{n \longrightarrow \infty} \frac{A_{n}\left(z^{-1}, \lambda\right)}{A_{n}\left(z^{-1},-\lambda\right)},
$$

where $z$ is the shifting operator and $T$ is the sampling period:

$$
\begin{aligned}
A_{o}\left(z^{-1}, \lambda\right) & =1, \\
A_{n}\left(z^{-1}, \lambda\right) & =A_{n-1}\left(z^{-1}, \lambda\right)-c_{n} z^{n} A_{n-1}(z, \lambda), \\
c_{n} & = \begin{cases}\frac{\lambda}{n}, & n \text { is odd } ; \\
0, & n \text { is even. }\end{cases}
\end{aligned}
$$

Consequently, the Laplace operator can be approximated to derive the FO integral and differential based on any given order of approximation $n$, as follows: 

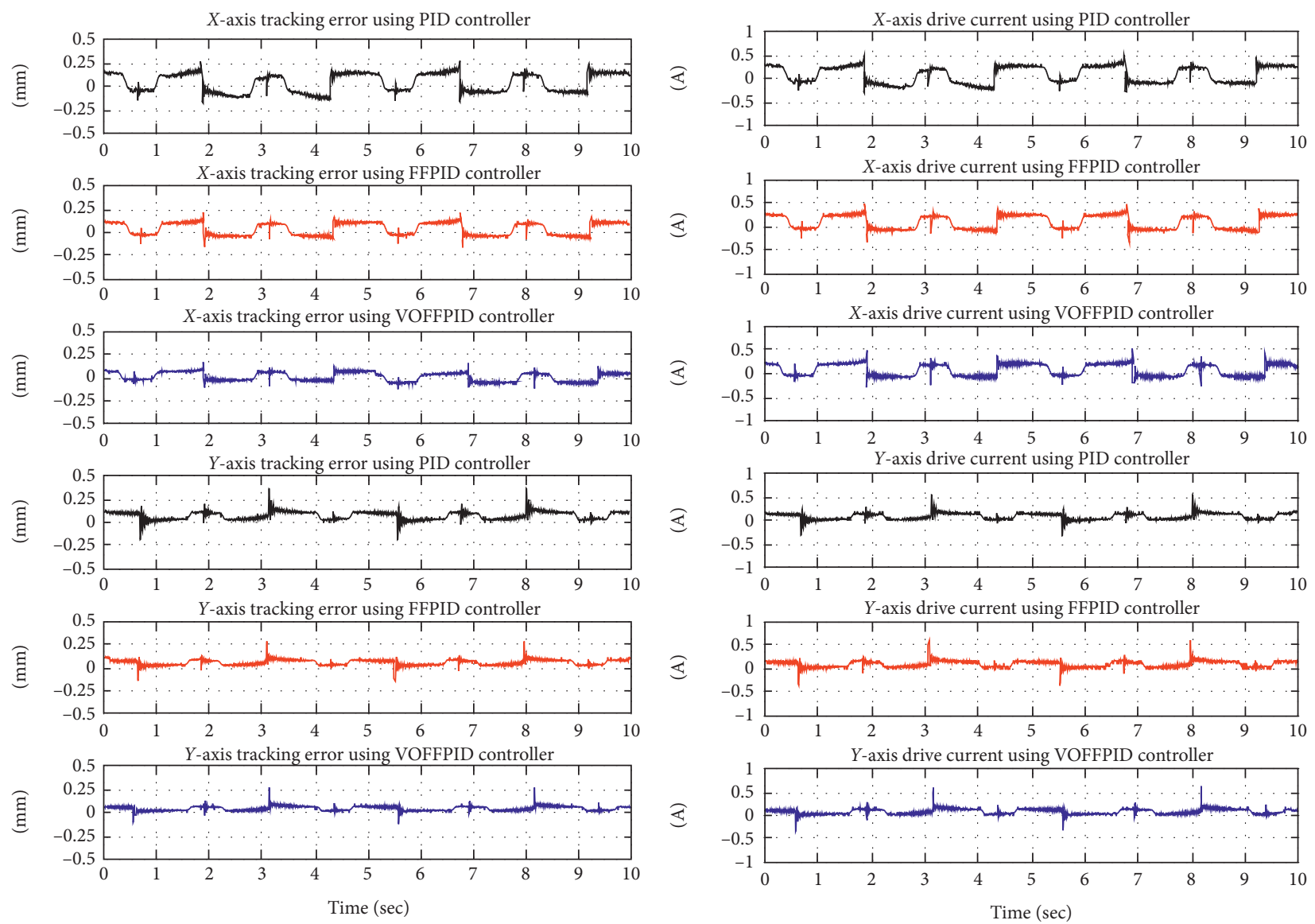

(a)

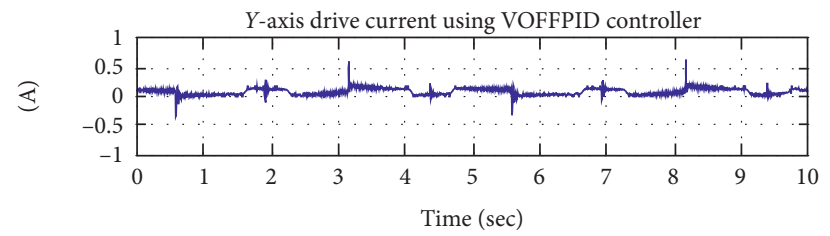

(b)

FIGURE 10: Flower contour tracking results of the VCMs-driven dual-axis positioning stage using PID, FFPID, and VOFFPID controllers in Case 2. (a) Tracking errors; (b) drive currents.

$$
s^{\lambda} \approx\left(\frac{2}{T}\right)^{\lambda} \frac{A_{n}\left(z^{-1}, \lambda\right)}{A_{n}\left(z^{-1},-\lambda\right)} .
$$

Thus, the FO operations can be realized via the digital implementation. Table 3 lists the expressions of $A_{n}\left(z^{-1}, \lambda\right)$ for $n=0,1,3$, and 5 .

5.3. Experimental Results. In the experiment, the control parameters of the PID controller were chosen as $K_{\mathrm{P} x}=25$, $K_{\mathrm{I} x}=110, K_{\mathrm{D} x}=10, K_{\mathrm{P} y}=25, K_{\mathrm{I} y}=110$, and $K_{\mathrm{D} y}=12$, respectively. Moreover, the control parameters of the FFPID controller are selected as $K_{\mathrm{P} x}=25, K_{\mathrm{I} x}=100, K_{\mathrm{D} x}=9, \lambda_{x}=1$, $a_{x}=0.6, \quad b_{x}=0.5, \quad K_{\mathrm{P} y}=25, \quad K_{\mathrm{I} y}=100, \quad K_{\mathrm{D} y}=11, \quad \lambda_{y}=1$, $a_{y}=0.5$, and $b_{y}=0.5$, respectively. In addition, a third-order approximation was used for the FO digital realization; that is, $n=3$. In this study, the control parameters were selected on the basis of several trials to achieve the favorable transient responses, considering the requirement of steady-state stability. However, designing an optimal set for all the control parameters is difficult because of the occurrence of uncertainties. Additionally, the PID and FFPID controllers cannot maintain ideal positioning performances by adopting the constant control parameters.
5.3.1. Flower Contour Tracking Results. The experimental results, including the tracking errors and drive currents of the VCMs-driven dual-axis positioning stage controlled by the PID, FFPID, and VOFFPID control systems due to the flower contour tracking in Cases 1 and 2, are shown in Figures 9 and 10, respectively. As can be seen from Figures 9(a) and 10(a), the mover of the stage can be successfully controlled by all the controllers to track the reference nonlinear contour shown in Figure 7. Furthermore, the drive currents in Case 2 are larger than those in Case 1, so a higher thrust force for the additional payload can be generated. The maximum tracking errors obtained in Case 1 for the PID, FFPID, and VOFFPID control systems were $0.2807 \mathrm{~mm}, 0.2363 \mathrm{~mm}$, and $0.1752 \mathrm{~mm}$, respectiely, whereas those obtained in Case 2 were $0.3973 \mathrm{~mm}, 0.2986 \mathrm{~mm}$, and $0.2731 \mathrm{~mm}$, respectively.

The tracking errors of the PID control system were unfavorable because of the large tracking errors. Although selecting larger control gains can diminish the amplutide of tracking errors, the excessive aggressive control gains may result in the oscillation of control responses.

As seen in Figures 9 and 10, the FFPID with two welldesigned variables $a$ and $b$ and FLS can derive more effective and smooth control signals to restrain the contouring errors 

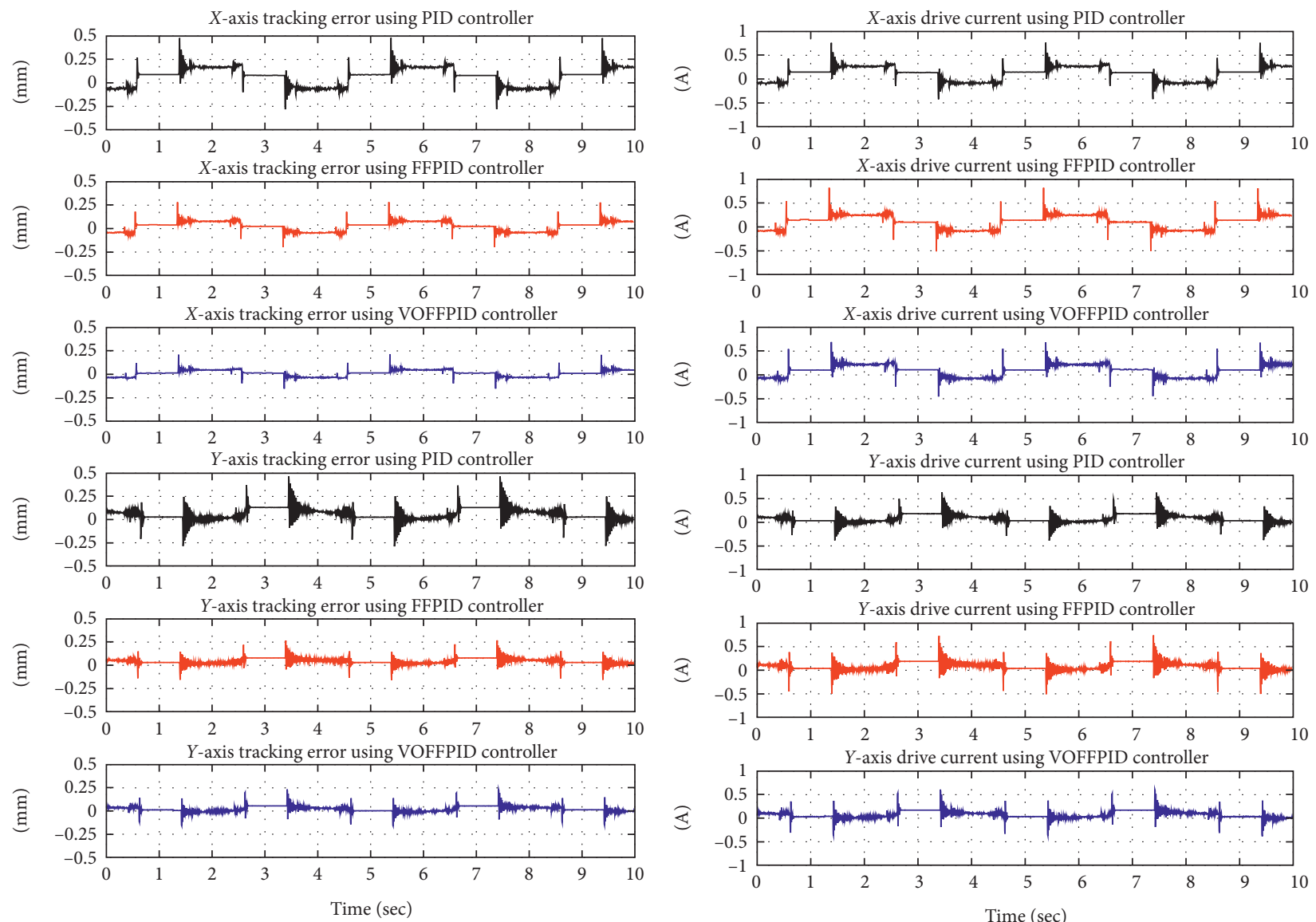

(a)

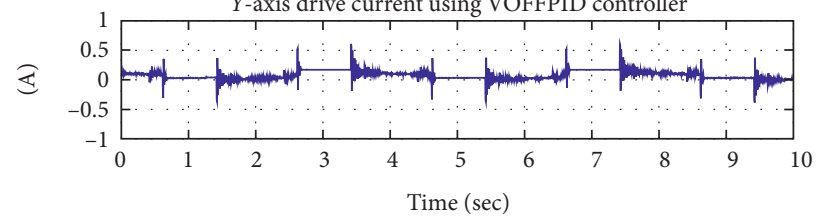

(b)

FIGURE 11: Window contour tracking results of the VCMs-driven dual-axis positioning stage using PID, FFPID, and VOFPID controllers in Case 1. (a) Tracking errors; (b) drive currents.

related to the specified dynamics of VCMs and the possible occurrence of uncertainties during the flower contour tracking. The corresponding tracking errors were reduced compared with those of the PID controller. Moreover, the FFPID controller has a good ability to diminish the effect of the disturbance, as shown in Figures 9(a) and 10(a). Although the control parameters of the FFPID controller were selected with several trials, the maximum and average tracking errors in the nominal and payload conditions are obviously reduced by the self-tuned strategy.

5.3.2. Window Contour Tracking Results. The experimental results due to the window contour tracking in Cases 1 and 2 are shown in Figures 11 and 12, respectively. Similar behaviors on the tracking responses of flower contour tracking can be observed. As seen from Figures 11(a) and 12(a), the maximum tracking errors obtained in Case 1 for the PID, FFPID, and VOFFPID control systems were $0.4877 \mathrm{~mm}$, $0.2843 \mathrm{~mm}$, and $0.2344 \mathrm{~mm}$, respectively, whereas those obtained in Case 2 were $0.5944 \mathrm{~mm}, 0.3512 \mathrm{~mm}$, and $0.3098 \mathrm{~mm}$, respectively. From the comparison in Figures 11(a) and 12(a), the tracking performances of the PID are evidently deteriorated when the contour command changes instantaneously. In contrast, the proposed VOFFPID demonstrates its robustness in the tracking performance during both test conditions. On the other hand, the control oscillations in the PID control system as shown in Figures 11(b) and 12(b) are evident due to its inefficient tracking ability. As opposed to the PID, more effective and smooth control signal was derived by the proposed VOFFPID to carry out the best control performance.

The experimental results and observations reveal that the optimized control parameters can improve the tracking performance in practical control applications. In Figures 9-12, the best control performance of the VOFFPID controller due to the flower and window contours under the nominal and payload conditions can be clearly observed. The improvement of the proposed VOFFPID controller in terms of the contour tracking accuracy is significant compared with that given by traditional PID and FFPID controllers.

The contour tracking performance measures of the PID, FFPID, and VOFFPID control systems for the tracking of the flower and window reference nonlinear contours are shown in Tables 4 and 5, respectively. They indicate that the FFPID controller with the integration of the PID control, FO operation, and FLS outperforms the conventional PID controller. Moreover, the proposed VOFFPID controller further 

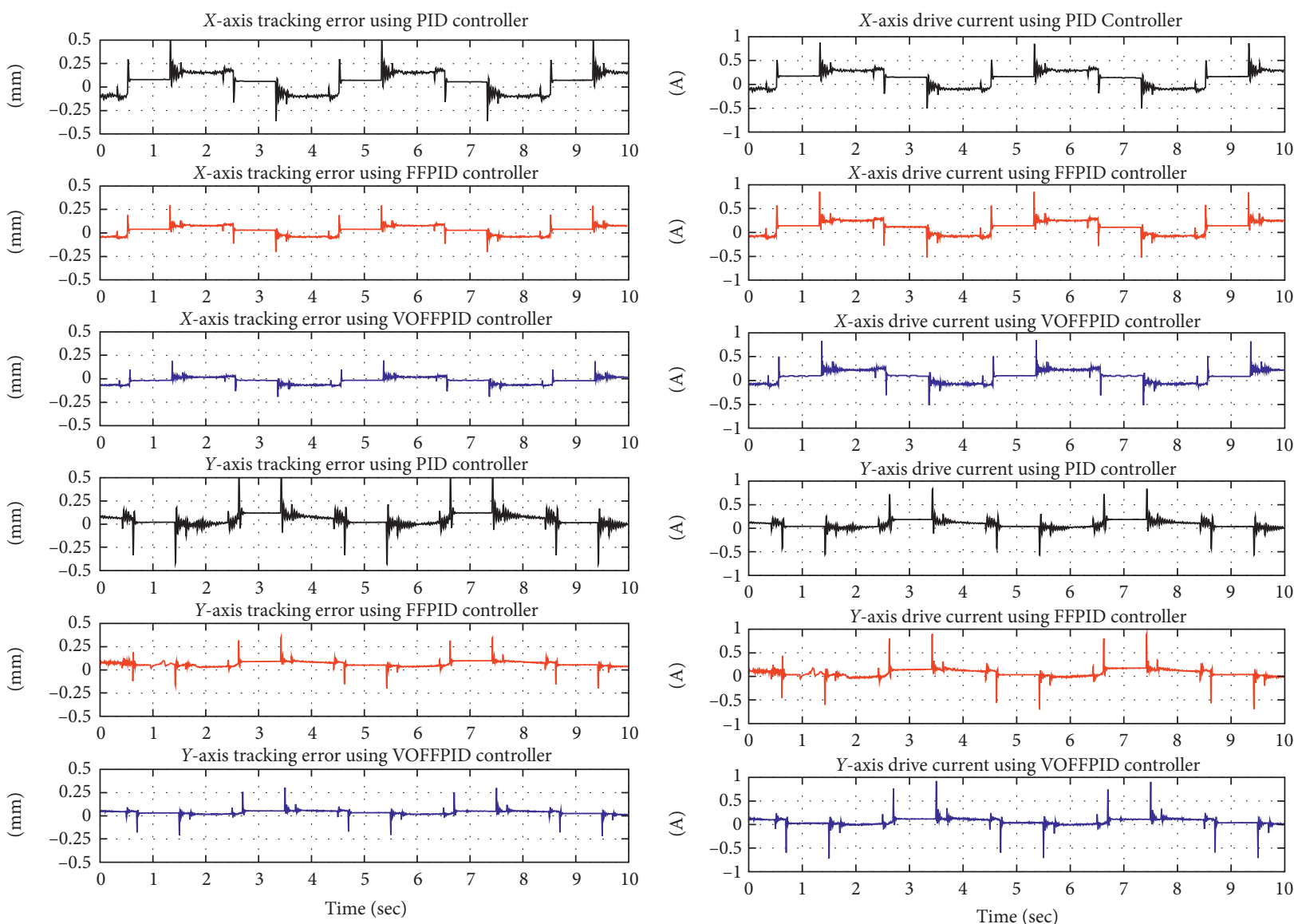

(a)

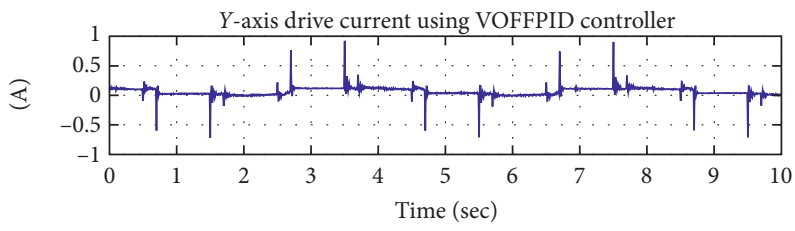

(b)

FIGURE 12: Window contour tracking results of the VCMs-driven dual-axis positioning stage using PID, FFPID, and VOFFPID controllers in Case 2. (a) Tracking errors; (b) drive currents.

TABLE 4: Contour tracking performance measures of Case 1.

\begin{tabular}{lccccr}
\hline & & & \multicolumn{2}{c}{ Commands } & \multicolumn{2}{c}{ Window contour (mm) } \\
Controllers & $T_{M}$ & Flower contour $(\mathrm{mm})$ & & $T_{M}$ & $T_{A}$ \\
\hline PID & 0.2807 & $T_{A}$ & $T_{S}$ & 0.4877 & 0.1389 \\
FFPID & 0.2363 & 0.1316 & 0.0336 & 0.2843 & 0.0756 \\
VOFFPID & 0.1752 & 0.0649 & 0.0233 & 0.2344 & 0.02655 \\
\hline
\end{tabular}

TABle 5: Contour tracking performance measures of Case 2.

\begin{tabular}{|c|c|c|c|c|c|c|}
\hline \multirow{3}{*}{ Controllers } & \multicolumn{6}{|c|}{ Commands } \\
\hline & \multicolumn{3}{|c|}{ Flower contour (mm) } & \multicolumn{3}{|c|}{ Window contour (mm) } \\
\hline & $T_{M}$ & $T_{A}$ & $T_{S}$ & $T_{M}$ & $T_{A}$ & $T_{S}$ \\
\hline PID & 0.3973 & 0.1312 & 0.0490 & 0.5944 & 0.1355 & 0.0597 \\
\hline FFPID & 0.2986 & 0.0921 & 0.0383 & 0.3512 & 0.0908 & 0.0253 \\
\hline VOFFPID & 0.2731 & 0.0682 & 0.0233 & 0.3098 & 0.0535 & 0.0289 \\
\hline
\end{tabular}

improves the tracking performance of the FFPID controller because all the control parameters were globally and dynamically optimized by the CSA algorithm. The VOFFPID controller apparently exhibits a high-precision contour tracking performance by effectively handling the payload and uncertainty during control processes. 


\section{Conclusions}

In this study, a VOFFPID control system is successfully developed and applied to control the mover position of a new VCMs-driven dual-axis positioning stage for tracking nonlinear reference contours. First, the structural and operating principles of the stage are introduced. Then, the CSA with the adaptive strategy for the optimization of control parameters is described. Subsequently, the theoretical bases of the PID, FFPID, and VOFFPID control systems are given in detail. With an additional degree of freedom to the control parameters and FLS operation, the FFPID controller can upgrade the contouring performances of the PID controller. Moreover, in the proposed VOFFPID controller, the CSA with the adaptive strategy can enhance the robustness of the FFPID controller by tuning the control parameters online. The experimental results subjected to different performance measures are given to verify the effectiveness of the proposed VOFFPID controller.

\section{Data Availability}

The experimental data used to support the findings of this study are included within the article.

\section{Conflicts of Interest}

The authors declare that they have no conflicts of interest.

\section{Acknowledgments}

The authors acknowledge the financial support of the Ministry of Science and Technology in Taiwan, R.O.C., under Grant no. MOST 108-2221-E-003-022-MY2.

\section{References}

[1] S. Das, I. Pan, S. Das, and A. Gupta, "A novel fractional order fuzzy PID controller and its optimal time domain tuning based on integral performance indices," Engineering Applications of Artificial Intelligence, vol. 25, no. 2, pp. 430-442, 2012.

[2] R. Sharma, P. Gaur, and A. P. Mittal, "Performance evaluation of cuckoo search algorithm based FOPID controllers applied to a robotic manipulator with actuator," in Proceedings of the 2015 International Conference on Advances in Computer Engineering and Applications, pp. 356-363, Ghaziabad, India, March 2015.

[3] Q. Sun, Z. Qi, J. Pei, and H. Liu, "Optimization of FOPID controller based on MPFGA," in Proceedings of the 2019 Chinese Control Conference, pp. 1846-1850, Guangzhou, China, July 2019.

[4] C. Osinski, G. Villar Leandro, and G. H. Da Costa Oliveira, "Fuzzy PID controller design for LFC in electric power systems," IEEE Latin America Transactions, vol. 17, no. 1, pp. 147-154, 2019.

[5] L. Xu, T. Xu, J. Wang, and X. Li, "A fuzzy PID controller-based two-axis compensation device for airborne laser scanning," IEEE Sensors Journal, vol. 17, no. 5, pp. 1353-1362, 2016.

[6] R. Sharma, K. P. S. Rana, and V. Kumar, "Performance analysis of fractional order fuzzy PID controllers applied to a robotic manipulator," Expert Systems with Applications, vol. 41, no. 9, pp. 4274-4289, 2014.

[7] S. Y. Chen, H. R. Lin, M. C. Yang, and Z. Y. Shen, "Fractionalorder fuzzy PID contouring control for a VCMs-based $X-Y$ motion stage," in Proceedings of the 2020 6th International Conference on Control, Automation and Robotics (ICCAR), pp. 236-241, Singapore, April 2020.

[8] I. S. Jesus and R. S. Barbosa, "Genetic optimization of fuzzy fractional PD + I controllers," ISA Transactions, vol. 57, pp. 220-230, 2015.

[9] T. Mahto and V. Mukherjee, "Fractional order fuzzy PID controller for wind energy-based hybrid power system using quasi-oppositional harmony search algorithm," IET Generation, Transmission \& Distribution, vol. 11, no. 13, pp. 3299-3309, 2017.

[10] L. Liu, S. Luo, F. Guo, and S. Tan, "Multi-point shortest path planning based on an improved discrete bat algorithm," Applied Soft Computing, vol. 95, Article ID 106498, 2020.

[11] X. Chen, B. Xu, C. Mei, Y. Ding, and K. Li, "Teachinglearning-based artificial bee colony for solar photovoltaic parameter estimation," Applied Energy, vol. 212, pp. 15781588, 2018.

[12] L. F. Grisales-Noreña, O. D. Montoya, and C. Andrés RamosPaja, "An energy management system for optimal operation of BSS in DC distributed generation environments based on a parallel PSO algorithm," Journal of Energy Storage, vol. 20, Article ID 101488, 2020.

[13] S. Gao, Y. Gao, Y. Zhang, and L. Xu, "Multi-strategy adaptive cuckoo search algorithm," IEEE Access, vol. 7, pp. 137642137655, 2019.

[14] Y. W. Zhang, L. Wang, and Q. D. Wu, "Dynamic adaptation cuckoo search algorithm," Control and Decision, vol. 29, no. 4, pp. 617-622, 2014.

[15] D. A. Nugraha, K. Lian, and Suwarno, "A Novel MPPT method based on cuckoo search algorithm and golden section search algorithm for partially shaded PV system," in Proceedings of the 2018 IEEE Electrical Power and Energy Conference (EPEC), pp. 1-6, Toronto, Canada, October 2018.

[16] A. Iglesias, A. Gálvez, P. Suárez et al., "Cuckoo search algorithm with Lévy flights for global-support parametric surface approximation in reverse engineering," Symmetry, vol. 10, no. 3, p. 58, 2018.

[17] S. Wu, Z. Jiao, L. Yan, R. Zhang, J. Yu, and C.-Y. Chen, "Development of a direct-drive servo valve with high-frequency voice coil motor and advanced digital controller," IEEE/ASME Transactions on Mechatronics, vol. 19, no. 3, pp. 932-942, 2014.

[18] S. Y. Chen and C. Y. Lee, "Digital signal processor based intelligent fractional-order sliding-mode control for a linear voice coil actuator," IET Control Theory \& Applications, vol. 11, no. 8, pp. 1282-1292, 2017.

[19] D. Huang, V. Venkataramanan, J.-X. Xu, and T. C. T. Huynh, "Contact-induced vibration in dual-stage hard disk drive servo systems and its compensator design," IEEE Transactions on Industrial Electronics, vol. 61, no. 8, pp. 4052-4060, 2014.

[20] S. Yabui, T. Atsumi, and T. Inoue, "Coupling controller design for MISO System of head positioning control systems in HDDs," IEEE Transactions on Magnetics, vol. 56, no. 5, pp. 1-9, 2020.

[21] C. E. Kim and Y. R. Kim, "Design and analysis of linear voice coil motor for automatic transmission," in Proceedings of the International Conference on Electrical Machines and Systems (ICEMS), College Station, TX, USA, February 2017.

[22] Y.-H. Chang, C.-S. Liu, I.-W. Chen, M.-S. Tsai, and H.-C. Tseng, "Open-loop control of voice coil motor with 
magnetic restoring force using high-low frequency composite signals," IEEE Access, vol. 7, pp. 146258-146263, 2019.

[23] K. J. Smith, D. J. Graham, and J. A. Neasham, "Design and optimization of a voice coil motor with a rotary actuator for an ultrasound scanner," IEEE Transactions on Industrial Electronics, vol. 62, no. 11, pp. 7073-7078, 2015.

[24] R. Wang, X. Yin, Q. Wang, and L. Jiang, "Direct amplitude control for voice coil motor on high frequency reciprocating rig," IEEE/ASME Transactions on Mechatronics, vol. 25, no. 3, pp. 1299-1309, 2020.

[25] S.-Y. Chen, T.-H. Li, and C.-H. Chang, "Intelligent fractionalorder backstepping control for an ironless linear synchronous motor with uncertain nonlinear dynamics," ISA Transactions, vol. 89, pp. 218-232, 2019.

[26] Akribis Systems Pte Ltd. AVM Series, http://www.akribis-sys. $\mathrm{com} /$.

[27] M. C. Yang, "Optimal fractional-order PID control for a VCMs-based X-Y motion stage," M.S. thesis, Department. Electric. Eng., National Taiwan Normal University, Taipei, Taiwan, 2020.

[28] M. Dulău, A. Gligor, and T. M. Dulău, "Fractional order controllers versus integer order controllers," Procedia Engineering, vol. 181, pp. 538-545, 2017.

[29] S. Ebrahimkhani, "Robust fractional order sliding mode control of doubly-fed induction generator (DFIG)-based wind turbines," ISA Transactions, vol. 63, pp. 343-354, 2016.

[30] G. Zhzo, "Fractional-order fast terminal sliding mode control for a class of dynamical systems," Mathematical Problems in Engineering, vol. 2013, Article ID 384921, 10 pages, 2013. 